\title{
Functional recovery despite prolonged bilateral loss of somatosensory evoked potentials: report on two patients
}

\author{
Hermann J Theilen, Maximilian Ragaller, Rüdiger von Kummer, Bernd Pohlmann-Eden,
} Gabriele Schackert, Michael D Albrecht

\begin{abstract}
A bilateral loss of short latency somatosensory evoked potentials (SSEPs) after head trauma or non-traumatic brain damage is normally associated with a deleterious neurological outcome. An adequate recovery in reported in two deeply comatose patients with head trauma or severe hypertensive encephalopathy despite prolonged bilateral loss of SSEPs over days, found in repeated recordings. Hence, a bilateral loss of SSEPs should not be considered alone for prediction of outcome in cerebral injury.

(F Neurol Neurosurg Psychiatry 2000;68:657-660)
\end{abstract}

Keywords: somatosensory evoked potentials; bilateral loss; outcome

Department of

Anaesthesiology and

Intensive Care

Medicine, Technical

University of Dresden,

University Hospital

Carl Gustav Carus,

Fetscherstraße 74,

01309 Dresden,

Germany

$\mathrm{H}$ J Theilen

M Ragaller

M D Albrecht

Department of

Neuroradiology

$\mathrm{R}$ von Kummer

Department of

Neurosurgery

G Schackert

Department Of

Neurology, Mannheim

Hospital of the

University of

Heidelberg, Theodor

Kutzer Ufer, 68135

Mannheim, Germany

B Pohlmann-Eden

Correspondence to:

Dr Hermann J Theilen

theilen@

rcs.urz.tu-dresden.de

Received 2 August 1999 and in final form

29 November 1999

Accepted 17 December 1999

Short latency somatosensory evoked potentials (SSEPs) provide important information on the integrity of the peripheral and central nervous system. A bilateral loss after head trauma or non-traumatic brain damage is associated with a deleterious neurological outcome, as stated in numerous studies. None the less, two authors described four selected adult patients with initially undetectable cortical responses showing a favourable outcome. ${ }^{12}$ After head trauma absent cortical responses were found in two patients. However, only single recordings were made within 48 hours after the insult. We found an adequate recovery in two deeply comatose patients with head trauma or severe hypertensive encephalopathy despite prolonged bilateral loss of SSEPs over days, found in repeated recordings.

\section{Clinical management}

The patients were admitted to an intensive care unit of a university hospital. Standard monitoring involved ECG, arterial blood pressure by peripheral arterial cannulation, arterial oxyhaemoglobin saturation by transcutaneous pulse oximetry, body temperature, intracranial pressure, and cerebral perfusion pressure. After admission to the intensive care unit, in both mechanically ventilated patients sedation was accomplished with intravenous midazolam $(1.3-7.4 \mu \mathrm{g} / \mathrm{kg} / \mathrm{min})$ and fentanyl (3.2-10.7 $\mathrm{ng} / \mathrm{kg} / \mathrm{min})$.
Brain stem auditory evoked potentials (BAEPs) and SSEPs were obtained using Nihon-Kohden Neuropack II TM. The SSEPs were elicited by repeated trials of electrical stimulation of median nerves at $5 \mathrm{~Hz}$ bilaterally. Median nerves were stimulated at the wrist until a visible thenar muscle twitch was found. Recording was performed at the second cervical level (N13 peak) and parietal scalp (N20 peak) with high grade steel needle electrodes. A total of 200 responses were averaged per trial. The BAEPs were elicited by $10 \mathrm{~Hz}$ alternating click stimulation of each ear (100 dB intensity) recorded at the vertex scalp with ipsilateral mastoid reference. A total of 1200 responses were averaged per trial.

\section{PATIENT 1}

After a car accident a 19 year old man was deeply comatose without any reaction to pain stimulus (Glasgow coma score 3). Both pupils were moderately dilated and promptly reactive to light. In addition to thoracic and abdominal injuries the patient had a traumatic subarachnoidal haemorrhage, brain contusions in both temporal hemispheres, and a generalised brain oedema. After emergency surgery which included the insertion of an epidural intracranial pressure (ICP) measuring device the patient was admitted to the intensive care unit. Haemodynamic stability was achieved by intravenous catecholamines. No signs of hypoxic events were documented until admission to the intensive care unit. On day 4 after admission an acute dilation of the right pupil accompanied by an rise in ICP from $20 \mathrm{~mm} \mathrm{Hg}$ up to $36 \mathrm{~mm} \mathrm{Hg}$ occurred. An immediate CT showed an increase of generalised brain oedema, which led to decompressive craniectomy on the right. This measure resulted in a constriction of the previously dilated pupil. Four hours after surgery recovery of pupillary responses to light was seen. After decompressive craniectomy ICP values stayed between 25 and $30 \mathrm{~mm} \mathrm{Hg}$ with a maximum value of 30 $\mathrm{mm} \mathrm{Hg}$ on day 3. Further ICP management consisted of osmodiuretic therapy with mannitol, and head elevation. The ICP decreased slowly to a normal range within 6 days after trauma. 
For a continuous measurement of jugular bulb oxygen saturation a catheter was inserted on the first day after admission. The lowest value during the 8 days after catheter insertion was $64 \%$. The lactate-oxygen index never exceeded 0.07 .

The first recording of SSEPs and BAEPs was performed on the second day after trauma. The BAEPs could not be elicited due to bilateral traumatic haemotympanum. A satisfactory recording was first achieved on day 22 after trauma and showed a substantial prolongation of I-V and III-V latencies.

A
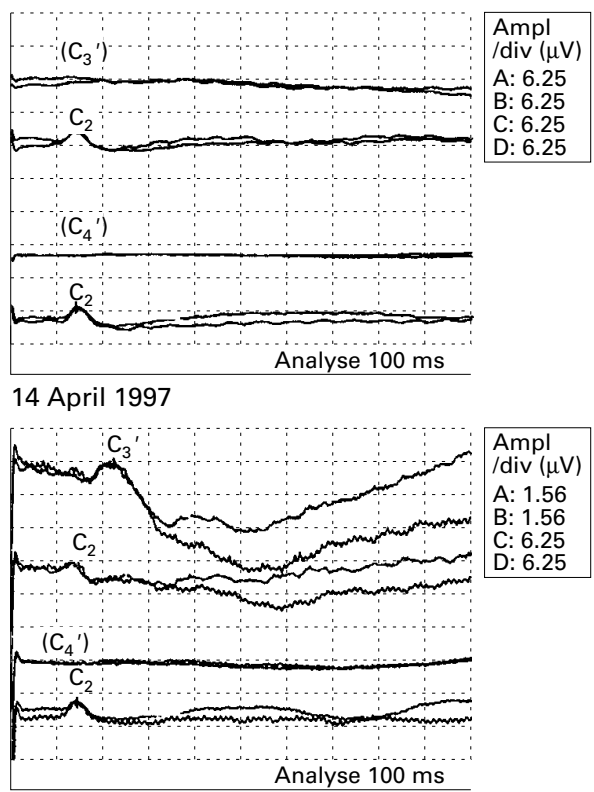

23 April 1997

B

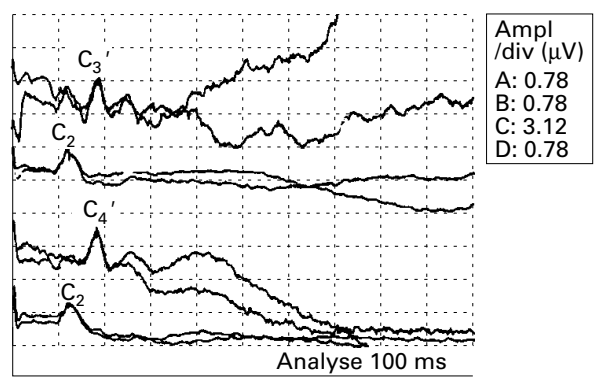

5 May 1997

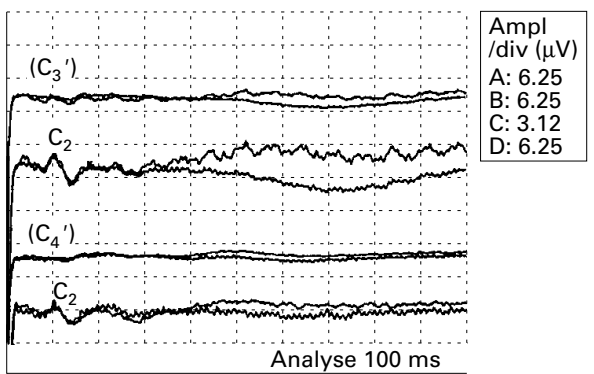

9 May 1997

Original median SSEP recordings of $(A)$ patient 1 and (B) patient 2. Time points of the recordings are given beneath each graph. Recordings of waves $N 20$ in left $\left(C_{3}{ }^{\prime}\right)$ and right hemisphere $\left(C_{4}{ }^{\prime}\right)$ are marked. $C_{2}$ describes the brain stem response at N13; duration of analysis was $100 \mathrm{~ms}$ in every graph; amplitude in $\mu$ Vldivision is displayed beside every response; if responses were found to be lost, the location of the expected cortical response is presented in parentheses.
For SSEPs, no cortical responses (N20/P25) could be detected whereas $\mathrm{N} 13$ responses were found for median nerve stimulation of both upper limbs. Another SSEP analysis 3 days later confirmed these findings. Nine days after trauma N20/P25-response in the left hemisphere recurred with a normal latency whereas in the right hemisphere the cortical response remained lost. On day 25 after trauma the right N20/P25-response could be detected although showing a strongly deformed complex and a pronounced prolongation of the central conduction time (13 ms, figure A).

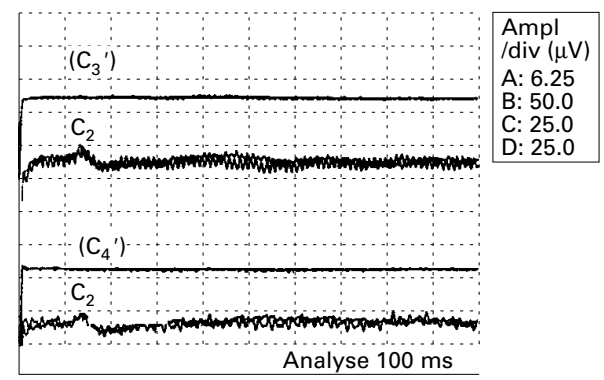

17 April 1997

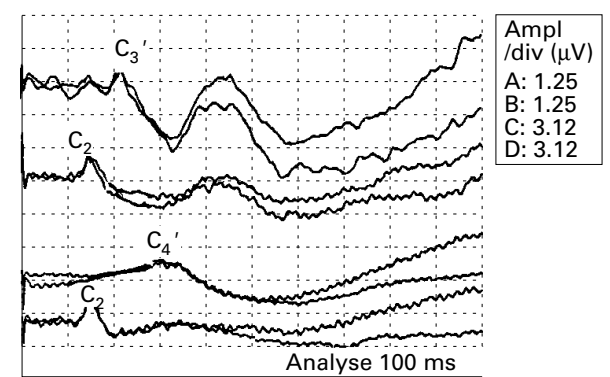

9 May 1997

7 May 1997

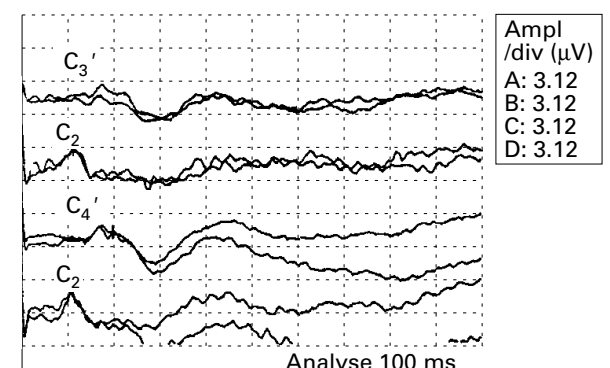

12 May 1997

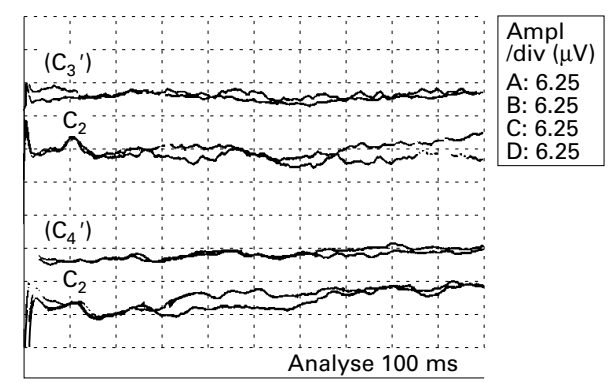


The EEG on the second day after trauma showed a predominant delta activity with occasionally detectable alpha activity. In an EEG on day 8 a frequency shift to theta activity was found and there were still severe posttraumatic alterations.

The clinical course showed a delayed arousal with the first signs of a repeatedly verifiable contact with the environment on day 15, 6 days after termination of sedation. On day 39 after trauma the patient was discharged from the intensive care unit and transferred to a rehabilitation centre. At discharge the patient was severely disabled. One year after trauma the patient is able, however, to live independently in his familiar surroundings, is able to speak, and to operate a computer device. Due to persisting incomplete hemiparesis on the left and behavioural deficits (emotional imbalance, impaired learning capacity) the current neurological state is described as moderately disabled.

PATIENT 2

A 30 year old woman developed eclampsia with generalised convulsive status epilepticus after 27 weeks of a previously uncomplicated pregnancy. Subsequently she became deeply comatose. After intubation and admission to hospital a cerebral CT and MRI showed multiple lesions in the supratentorial and infratentorial brain regions in combination with a single focus of intracerebral haemorrhage in the right hemisphere which were interpreted as infarctions. An epidural device for ICP monitoring was inserted surgically. The patient was transferred to the intensive care unit and treated as described above. On the second day after admission delivery was performed by caesarean section.

The ICP monitoring showed pathological values during the first 3 days after admission with a maximum at $24 \mathrm{~mm} \mathrm{Hg}$. After delivery the ICP dropped to values within the upper normal range. Sedation was stopped as cerebral CT on day 4 after caesarean section showed a declining perifocal oedema in the vicinity of the persisting infarcted areas and she did not show signs of a critically raised intracranial pressure. Four days later the patient regained consciousness and was extubated. Brain MRI showed no signs of cerebral oedema 12 days after admission; merely a small remnant of the initially described intracerebral haemorrhage was visible. According to these findings cerebral pathology was diagnosed as a severe hypertensive encephalopathy, which can be seen often in severe eclamptic syndrome. One year after admission the patient showed complete neurological recovery.

Her SSEPs and BAEPs were studied on days $2,4,6$, and 9 after admission to the intensive care unit. On day 2 both cortical responses were found whereas a bilateral loss of N20/P25 response was found on days 4 and 6 with still detectable responses at N13. On day 9 $\mathrm{N} 20 / \mathrm{P} 25$ waves recovered in both hemispheres (figure B). The BAEPs showed an inconsiderable prolongation of I-V latencies in all record- ings obtained during her stay in the intensive care unit.

The EEG on day 2 after admission presented predominantly with theta/delta activity with occasionally occurring sleep spindle activity. After showing a further frequency decrease on day 4, normalisation was observed on day 6 with predominant alpha activity, which could be ascribed to a still persisting effect of sedative drugs.

\section{Discussion}

We have shown that bilaterally abolished short latency SSEPs seen in adults early after brain injury and which persist for days do not inevitably result in a poor neurological outcome.

We think that we can exclude the possibility that technical failure caused the loss of SSEP in our patients. All recordings were performed by an experienced investigator. Electrodes were placed precisely in accordance with international standards. Neuronal lesions in the peripheral nervous system or the spinal cord could be ruled out as a brain stem response at $\mathrm{N} 13$ was found in both patients. This finding eliminates the possibility of technical problems in SSEP generation.

A disappearance of SSEPs in both hemispheres after brain injury implies a high probability of a poor neuropsychological outcome, in particular in adults, and after hypoxic brain damage. ${ }^{3}$ This phenomenon could be due to a disruption of the somatosensory pathway (neuronal or axonal injury) or a breakdown of cell membrane integrity caused by substantial energy depletion (ischaemia or hypoglycaemia). Theoretically, a reversibility of the pathophysiological process exists for both events although axonal or neuronal injury of the cerebrum often results in irreversible neuronal cell death. ${ }^{4}$

The extent of cortical or subcortical functional disorder due to derangement of cerebral oxidative metabolism depends on the extent and duration of energy deficiency. Experimental data showed that after complete abolition of SSEPs after 2 hours of regional brain ischaemia a restoration of blood supply resulted in a reactivation of cortical responses. ${ }^{5-7}$ Even in the clinical situation, a complete loss of SSEPs induced by carotid clamping is not inevitably combined with irreversible brain damage. ${ }^{8}$ Translated to the situation of brain traumatised patients a transient brain ischaemia could induce a reversible bilateral loss of SSEPs if blood flow is restored in an acceptable time. Bouma et $a l^{9}$ found a substantial derangement of blood supply especially in the first 4 hours after trauma. According to these findings it can be hypothesised that a bilateral loss of SSEPs during the first hours after trauma might be unreliable for prediction of outcome. A rapid and sufficient restoration of cerebral oxygen supply prevents irreversible brain damage, thus inducing a restoration of previously absent SSEPs. This might explain the phenomenon of restored SSEPs in a patient with head trauma described by Schwarz et al, ${ }^{2}$ who showed lost SSEPs 3 hours after head trauma with restoration 24 hours later. The fact that in our patients 
a bilateral loss of SSEPs was observed in repeated recordings over several days excludes abolished SSEPs due to ischaemia.

The prolonged loss of SSEPs can be interpreted as a compromised morphology of the somatosensory pathway thus leading to selective reversible blockade of the ascending somatosensory pathway. A mechanical lesion due to axonal or neuronal compression in the CNS by transient transtentorial herniation was assumed by Schwarz et al for three of their four patients. By contrast, in our patients pupillary abnormalities, if seen, were rapidly reversible. Although SSEPs were abolished, pupillary responses presented normally, which excludes the idea of Schwarz et al in our patients.

Pathophysiological explanations for the reappearance of SSEPs remain hypothetical. It can only be assumed that reversible structural and functional derangement in the somatosensory pathway produced a transient blockade of neuronal transmission. In patient 1 the right sided pathological alteration of the cortical response persisting 4 weeks after trauma is obviously indicative of a focal hemispheric cerebral deficit.

In conclusion, based on two findings only we cannot in principle doubt the highly predictive value of SSEPs in patients with brain injury. A bilateral loss of SSEPs, however, should not be considered alone for outcome prediction and the decisions on further therapeutic strategy in cerebral injury.

1 Lindsay K, Pasaoglu A, Hirst D, et al. Somatosensory and auditory brain stem conduction after head injury. a comparison with clinical features in prediction of outcome. Neurosurgery 1990;26:278-85.

2 Schwarz S, Schwab S, Aschoff A, et al. Favorable recovery from bilateral loss of somatosensory evoked potentials. Crit Care Med 1999;27:182-7.

3 Pohlmann-Eden B, Dingethal K, Bender HJ, et al. How reliable is the predictive value of SEP (somatosensory evoked potentials) patterns in severe brain damage with special regard to the bilateral loss of cortical responses? Intensive Care Med 1997;23:301-8.

4 Barron KD. Axon reaction and its relevance to CNS trauma. In: RG Grossman, PL Gildenberg, eds. Head injury: basic and clinical aspects. New York: Raven Press 1982:45-55.

5 Graf R, Kataoka K, Rosner G, et al. Cortical deafferentation in cat focal ischaemia: disturbance and recovery of sensory functions in cortical areas with different degrees of cerebral blood flow reduction. I Cereb Blood Flow Metab 1986;6: 566-73.

6 Kataoka K, Graf R, Rosner G, et al. Experimental focal ischemia in cats: changes in mulitmodality evoked potentials to local cerebral blood flow and ischemia. Stroke 1987; 18:188-94.

7 Mizoi K, Suzuki H, Abiko H, et al. Experimental study on the reversibility of cerebral ischemia: residual blood flow and duration of ischemia. Acta Neurochir (Wein) 1987;88: $126-34$.

8 Beese U, Langer H, Lang W, et al. Comparison of near infrared spectroscopy and somatosensory evoked potentials for the detection of cerebral ischemia during carotid endarterectomy. Stroke 1998;29:2032-7.

9 Bouma GJ, Muizelaar JP. Cerebral circulation and metabolism after severe traumatic brain injury: the elusive role of ischemia. $\mathcal{F}$ Neurosurg 1991;75:685-93. 